\title{
Associação entre paracoccidioidomicose e alcoolismo
}

\section{The relationship between paracoccidioidomycosis and alcoholism}

\author{
Roberto Martinez", Maria Janete Moya"
}

\begin{abstract}
MARTINEZ, R. \& MOYA, M.J. Asseciaçāo entre paracoccidioidomicose e alcoolismo. Rev. Sauide públ., S.Paulo, 26: 12 - 6, 1992 A associação entre alcoolismo e paracoccidioidomicose foi avaliada pelo método de caso-controle, comparando-se o hábito de ingestão etílica de 70 doentes com o de outros 70 pacientes hospitalizados por razões diversas e pareados por sexo e idade. Os participantes foram interrogados de maneira padronizada sobre a quantidade, tipo e periodicidade da ingestảo de bebidas alcoólicas, duração do consumo e também sobre manifestações de abuso e/ou dependència do álcool. $\mathrm{Na}$ forma crônica da micose foi observada proporção significativamente maior de doentes com ingestão média de álcool acima de $60 \mathrm{ml} / \mathrm{dia}(50,0 \% \mathrm{x}$ $30,0 \%)$ e com preferència por aguardente de cana $(89,4 \% \times 68,3 \%)$ em relação ao grupo controle, além dos grandes bebedores (>100 ml/dia) mostrarem tendência de reativação da doença durante ou após seu tratamento. $\mathrm{Na}$ forma aguda/subaguda da paracoccidioidomicose verificou-se que 64,3\% dos consumidores de bebidas alcoólicas já haviam tido um ou mais episódios de embriaguez etílica, versus $17,6 \%$ no grupo controle. Os dados sugerem que o alcoolismo seja fator predisponente da paracoccidioidomicose e talvez possa prejudicar sua cura, principalmente da forma crônica da infecção.
\end{abstract}

Descritores: Alcoolismo, complicaçōes. Paracoccidioidomicose, complicações.

\section{Introdução}

A paracoccidioidomicose afeta predominantemente trabalhadores rurais da América Latina, muitos dos quais ingerem bebidas alcoólicas com regularidade ${ }^{6}$. $O$ etilismo foi considerado possível fator predisponente da micose por ser encontrado em mais de $50 \%$ dos pacientes de uma casuística de São Paulo - Brasil ${ }^{4}$. Em razão de coexistirem nas áreas endêmicas de paracoccidioidomicose, sugeriu-se que alcoolismo e desnutrição estariam envolvidos na patogenia dessa doença ${ }^{10}$.

Não se encontrando relato de estudo que confirmasse a associação entre paracoccidioidomicose e alcoolismo, recorreu-se ao método de caso controle para investigar o consumo excessivo de álcool como fator de risco e de má evolução da infecção fúngica. Com esta forma de investigação demonstrou-se anteriormente a asșociação entre alcoolismo e tuberculose pulmonar.

\section{Material e Método}

Constituíram os casos do estudo 70 doentes

* Departamento de Clínica Médica da Faculdade de Medicina de Ribeirão Preto da Universidade de São Paulo - Ribeirăo Preto, SP - Brasil.

** Departamento de Clínica Médica do Hospital das Clínicas da Faculdade de Medicina de Ribeirão Preto da Universidade de São Paulo - Ribeirão Preto, SP - Brasil. Separatas/Reprints: R.Martinez - Av. Bandeirantes, 3900 14049 - Ribeirão Preto, SP - Brasil.

Publicação financiada pela FAPESP. Processo Saúde Coletiva 91/4994-0. de paracoccidioidomicose assistidos no Hospital das Clínicas da Faculdade de Medicina de Ribeirão Preto - Estado de São Paulo, no período entre setembro de 1986 e julho de 1988 . O diagnóstico foi confirmado pelo isolamento do $P$. brasiliensis em cultura, por exame histopatológico e ou exame sorológico. Foram subdivididos em dois grupos, de acordo com a idade e quadro clínico apresentado durante a fase ativa da moléstia e conforme classificação adotada atualmente ${ }^{11}$ : forma aguda/subaguda (Pcm aguda) e forma crônica (Pcm crônica). Formaram o grupo Pcm aguda 20 doentes, 17 do sexo masculino e 3 do feminino, faixa etária dos 16 aos 48 anos (média: 27,4 anos) e com lesão predominante do sistema linfático. $O$ grupo $\mathrm{Pcm}$ crônica ficou formado por 50 doentes, 47 do sexo masculino e 3 do feminino, faixa etária dos 29 aos 77 anos (média: 50,9 anos), a maioria com lesão pulmonar. Os doentes de paracoccidioidomicose foram comparados com outros 70 pacientes do Hospital das Clínicas, das enfermarias de Ortopedia, Oftalmologia, Dermatologia e Clínica Médica. Utilizando a metodologia de caso-controle ${ }^{9}$, estes pacientes foram pareados individualmente, por sexo e idade, com os doentes de paracoccidioidomicose, constituindo-se então dois grupos controles:

Controle-A (20 pacientes) e Controle-C (50 pacientes), correspondentes aos grupos $\mathrm{Pcm}$ aguda e Pcm crônica, respectivamente.

Os 70 casos e os 70 controles foram entrevistados, de maneira padronizada, por uma única pessoa que formulou questões consecutivas so- 
bre: a) hábito de ingestão de bebidas alcoólicas anteriormente ao surgimento da infecção fúngica; b) tipos de bebidas ingeridas; c) duração do hábito; d) frequência da ingestão (diária, semanal ou menos frequente) e periodos do dia em que eram consumidas as bebidas; e) quantidade ingerida de cada tipo de bebida (no dia, na semana ou no mês); f) se o paciente havia chegado ao estado de embriaguez (alteração do comportamento por efeito do álcool) e a frequência com que isto acontecia; g) se o paciente sentia-se mal caso não tomasse bebidas alcoólicas (necessidade de ingestão destas bebidas). A ficha de cada doente de paracoccidioidomicose foi completada com dados sobre a moléstia: data do início, localização das lesões, classificação e evolução da infecção depois de instituído o tratamento. A evolução foi considerada boa quando havia cura aparente em seguimento minimo de um ano e má quando apresentavam um ou mais episódios de reativação da doença. Não foi possivel conhecer a evolução da doença em 5 pacientes que abandonaram o seguimento (um com a forma $\mathrm{Pcm}$ aguda e $4 \mathrm{com}$ a forma $\mathrm{Pcm}$ crônica).

Para o cálculo do consumo diário de álcool utilizou-se a concentração usual do mesmo nas diferentes bebidas ingeridas: aguardente de cana $40 \%$; cerveja - $3 \%$; vinho - $11 \%$; outras bebidas destiladas - entre $38 \%$ e $45 \%$, conforme o tipo. $\mathrm{Na}$ comparação entre os grupos de pacientes considerou-se ingestão superior a $60 \mathrm{ml}(50 \mathrm{~g})$ por dia como correspondente a consumo significativo de álcool $^{20}$ e suficiente para predispor a infecçōes ${ }^{12}$.

$\mathrm{O}$ teste do quiquadrado, o teste exato de Fisher ${ }^{5}$ e o teste $t$ de Student ${ }^{7}$ foram os métodos estatísticos empregados, tomando-se alfa $=5 \%$ como nivel de significância.

\section{Resultados}

\section{Quantidade de álcool ingerido}

A maioria dos individuos interrogados ingeria bebidas alcoólicas em quantidade variando aproximadamente entre $1 \mathrm{ml} \mathrm{a} 600 \mathrm{ml}$ de álcool por dia (Tabela 1). $O$ consumo foi mais elevado no gnupo de doentes com paracoccidioidomicose crônica, embora a diferença com a média do grupo Con-
Tabela 2. Distribuição dos doentes de paracoccidioidomicose aguda ou crônica e respectivos grupos controle segundo o consumo diário médio de álcool. Ri. beirāo Preto - SP, Brasil, 1986-1988

\begin{tabular}{|c|c|c|c|c|c|c|}
\hline \multirow{3}{*}{ Grupo Clínico } & \multicolumn{4}{|c|}{$\begin{array}{r}\text { Consumo de } \\
\text { álcool (ml/dia) }\end{array}$} & \multicolumn{2}{|c|}{ Total } \\
\hline & \multicolumn{2}{|c|}{0.60} & \multicolumn{2}{|c|}{$>60$} & \multirow[t]{2}{*}{$n^{e}$} & \multirow[t]{2}{*}{$\%$} \\
\hline & $n^{2}$ & $\%$ & $n^{2}$ & $\%$ & & \\
\hline $\begin{array}{l}\text { Paracoccidioidomicose } \\
\text { aguda }\end{array}$ & 18 & 90,0 & 2 & 10,0 & 20 & 100,0 \\
\hline Grupo Controle A & 17 & 85,0 & 3 & 15,0 & 20 & 100,0 \\
\hline Total & 35 & & 5 & & 40 & \\
\hline $\begin{array}{l}\text { Paracoccidioidomicose } \\
\text { crônica }\end{array}$ & 25 & 50,0 & 25 & $50,0^{\circ}$ & 50 & 100,0 \\
\hline Grupo Controle C & 35 & 70,0 & 15 & $30,0^{\circ}$ & 50 & 100,0 \\
\hline Total & 60 & & 40 & & 100 & \\
\hline
\end{tabular}

$p<0,05\left(x^{2}=4,167\right)$

trole-C não alcançasse significado estatístico $(t=$ $1,476, \mathrm{p}>0,05$ ).

Bebiam além de $60 \mathrm{ml}$ por dia, respectivamente, $10 \%$ e $15 \%$ dos pacientes dos grupos $\mathrm{Pcm}$ aguda e Controle-A (Tabela 2). No grupo Pcm crônica foi encontrada proporção significativamente maior de pacientes com ingestão alcoólica superior a $60 \mathrm{ml} / \mathrm{dia}(50,0 \%)$ do que no correspondente Controle-C $(30,0 \%)\left(x^{2}=4,167, p<0,05\right)$.

\section{Periodicidade da ingestão alcoólica}

A frequência de consumo de todos que tomavam bebidas alcoólicas foi classificada em diária e não diária. No grupo $\mathrm{Pcm}$ aguda predominou o consumo não diário $(64,3 \%)$, o mesmo ocorrendo no Controle-A $(76,5 \%)$. O consumo diário predominou no grupo Pcm crônica $(76,6 \%)$ e no Controle-C $(61,9 \%)$. As diferenças entre os grupos não são significativas.

\section{Tipo de bebida alcoólica}

A cada paciente que referiu tomar bebidas alcoólicas, interrogou-se qual era ingerida preferencialmente. Todos consumiam maior quantidade de aguardente de cana ou de cerveja, exceto um doente com Pcm aguda que ingeria mais vinho e um paciente do grupo Controle-C que ingeria mais co-

Tabela 1. Consumo de álcool por doentes com paracoccidioidomicose $(\mathrm{Pcm})$ aguda ou crônica e respectivos grupos controles. Ribeirão Preto - SP, Brasil, 1986-1988

\begin{tabular}{lcccccc}
\hline Grupo de & \multicolumn{2}{c}{ Ingestăo de bebidas alcoólicas $(n)$} & \multicolumn{3}{c}{ Consumo diário de álcool (ml) } \\
\cline { 2 - 7 } pacientes & Não & Sim & Total & Média \pm DP & Mediana & Variação \\
\hline Pcm aguda & 6 & 14 & 20 & $24,3 \pm 26,5$ & 8,1 & $0,0 \cdot 73,9$ \\
Controle A & 3 & 17 & 20 & $27,7 \pm 62,3$ & 5,2 & $0,0 \cdot 258,0$ \\
Pcm crônica & 3 & 47 & 50 & $95,0 \pm 121,1$ & 58,9 & $0,0 \cdot 600,2$ \\
Controle C & 8 & 42 & 50 & $61,3 \pm 106,9$ & 22,7 & $0,0-485,0$ \\
\hline
\end{tabular}

$\mathrm{n}$ = tamanho da amostra; DP = desvio padrão 
Tabela 3. Tipo de bebida alcoólica ingerida preferencialmente segundo o grupo de pacientes. Ribeirão Preto - SP, Brasil, 1986-1988.

\begin{tabular}{lccccccc}
\hline \multirow{2}{*}{ Grupo Clinico } & \multicolumn{3}{c}{ Tipo de bebida } & \multicolumn{2}{c}{ Total } \\
\cline { 2 - 6 } & \multicolumn{2}{c}{ Aguardente } & Cerveja & $n^{2}$ & $\%$ \\
\cline { 2 - 6 } & $n^{2}$ & $\%$ & $n^{2}$ & $\%$ & & \\
\hline $\begin{array}{l}\text { Paracoccidioidomicose } \\
\text { aguda }\end{array}$ & 4 & 30,8 & 9 & 69,2 & 13 & 100,0 \\
Grupo Controle A & 8 & 47,1 & 9 & 52,9 & 17 & 100,0 \\
Total & 12 & & 18 & & 30 & \\
$\begin{array}{l}\text { Paracoccidioidomicose } \\
\text { crónica }\end{array}$ & 42 & $89,4^{*}$ & 5 & 10,6 & 47 & 100,0 \\
Grupo Controle C & 28 & $68,3^{*}$ & 13 & 31,7 & 41 & 100,0 \\
Total & 70 & & 18 & & 88 & \\
\hline$p<0,025\left(x^{2}=5,974\right)$ & & & & &
\end{tabular}

nhaque. Estes dois casos não foram considerados na análise feita na Tabela 3 , onde se observa ingestão predominante de cerveja nos grupos $\mathrm{Pcm}$ aguda $(69,2 \%)$ e Controle-A $(52,9 \%)$ e de aguardente de cana nos grupos Pcm crônica $(89,4 \%)$ e Controle-C $(68,3 \%)$. A maior ingestão de aguardente no grupo $\mathrm{Pcm}$ crônica em relação ao controle-C é estatisticamente significante $\left(x^{2}=5,974\right.$, $\mathrm{p}<0,025)$.

Tabela 4. Ocorrência de episódio prévio de embria. guez etilica, segundo o grupo clínico, em pacientes que ingeriam bebidas alcoólicas. Ribeirão Preto - SP, Brasil, 1986-1988

\begin{tabular}{|c|c|c|c|c|c|c|}
\hline \multirow{3}{*}{ Grupo Clínico } & \multicolumn{4}{|c|}{ Embriaguez prévia } & \multicolumn{2}{|c|}{ Total } \\
\hline & \multicolumn{2}{|c|}{ Sim } & \multicolumn{2}{|c|}{ Não } & \multirow[t]{2}{*}{$n^{2}$} & \multirow[t]{2}{*}{$\%$} \\
\hline & $n^{2}$ & $\%$ & $n^{2}$ & $\%$ & & \\
\hline $\begin{array}{l}\text { Paracoccidioidomicose } \\
\text { aguda }\end{array}$ & 9 & $64,3^{*}$ & 5 & 35,7 & 14 & 100,0 \\
\hline Grupo Controle A & 3 & $17,6^{\circ}$ & 14 & 82,4 & 17 & 100,0 \\
\hline Total & 12 & & 19 & & 31 & \\
\hline $\begin{array}{l}\text { Paracoccidioidomicose } \\
\text { crónica }\end{array}$ & 17 & 36,2 & 30 & 63,8 & 47 & 100,0 \\
\hline Grupo Controle C & 10 & 23,8 & 32 & 76,2 & 42 & 100,0 \\
\hline Total & 27 & & 62 & & 89 & \\
\hline
\end{tabular}

\section{Estado de embriaguez}

Dentre os consumidores de bebidas alcoólicas foram identificados os que haviam chegado ao estado de embriaguez em pelo menos uma ocasião. Isto ocorrera com $64,3 \%$ dos casos no grupo $\mathrm{Pcm}$ aguda, contra $17,6 \%$ no Controle-A, diferença significante $\left(x^{2}=7,039, p<0,01\right)$. Nos grupos Pcm crônica e Controle-C já haviam se embriagado $36,2 \%$ e $23,8 \%$, respectivamente, dos indivíduos que ingeriam álcool, diferença não significativa (Tabela 4).

\section{Necessidade de ingestão do álcool}

Alegaram que ingestão de álcool era necessária para seu bem-estar psíquico ou orgânico, $0,0 \%$ dos casos no grupo Pcm aguda, $17,7 \%$ no Controle-A, $17,0 \%$ no grupo Pcm crônica e $14,3 \%$ no Controle-C. Não se encontrou diferença entre os grupos em comparação.

\section{Duração do hábito de ingestāo de bebidas al- coólicas}

O valor mediano para o período prévio de consumo de bebidas alcoólicas nos grupos Pcm aguda e Controle-A correspondeu a 9,5 e 10,0 anos, e nos grupos Pcm crônica e Controle-C correspondeu a 28,0 e 24,0 anos (diferenças não significativas).

Evolução após tratamento da paracoccidioidomicose

Foram observados durante período mínimo de 1 ano de tratamento 19 doentes do grupo $\mathrm{Pcm}$ aguda e 46 doentes com Pcm crônica, relacionando-se o tipo de evolução com o consumo diário médio de álcool (Tabela 5). Não são significativas as diferenças entre as proporções obtidas $\left(x^{2}=3,681\right)$, mas destaca-se a tendência à má evolução dos doentes crônicos que ingeriam mais de $100 \mathrm{ml}$ de álcool por dia. Dos 5 casos que abandonaram o seguimento, 4 eram do grupo Pcm crônica e tinham consumo alcoólico diário de, respectivamente, $10,4 \mathrm{ml}, 90,0 \mathrm{ml}, 123,6 \mathrm{ml} \mathrm{e} 600,2 \mathrm{ml}$; um era do grupo $\mathrm{Pcm}$ aguda e não ingeria álcool.

Tabela 5. Evolução dos doentes de paracoccidioidomicose após tratamento, segundo a forma clínica e a ingestāo diária média de álcool. Ribeirāo Preto - SP, Brasil, 1986 - 1988

\begin{tabular}{|c|c|c|c|c|c|c|c|c|c|c|c|c|}
\hline \multirow{3}{*}{$\begin{array}{l}\text { Ingestão } \\
\text { de álcool } \\
\mathrm{ml} / \mathrm{dia}\end{array}$} & \multicolumn{6}{|c|}{ Forma aguda - evolução } & \multicolumn{6}{|c|}{ Forma crônica - evolução } \\
\hline & \multicolumn{2}{|c|}{ Boa } & \multicolumn{2}{|c|}{ Má } & \multicolumn{2}{|c|}{ Total } & \multicolumn{2}{|c|}{ Boa } & \multicolumn{2}{|c|}{ Má } & \multicolumn{2}{|c|}{ Total } \\
\hline & $n^{2}$ & $\%$ & $n^{2}$ & $\%$ & $n^{2}$ & $\%$ & $n^{2}$ & $\%$ & $n^{2}$ & $\%$ & $n^{2}$ & $\%$ \\
\hline $0 \cdot 60$ & 9 & 52,9 & 8 & 47,1 & 17 & 100,0 & 16 & 66,7 & 8 & 33,3 & 24 & 100,0 \\
\hline $61 \cdot 100$ & 2 & 100,0 & 0 & 0,0 & 2 & 100,0 & 6 & 60,0 & 4 & 40,0 & 10 & 100,0 \\
\hline$>100$ & 0 & & 0 & & 0 & & 4 & 33,3 & 8 & 66,7 & 12 & 100,0 \\
\hline Total & 11 & & 8 & & 19 & & 26 & & 20 & & 46 & \\
\hline
\end{tabular}




\section{Discussão}

A ingestão de grande quantidade de bebidas alcoólicas foi referida por parcela considerável dos entrevistados de maior faixa etária, confirmando a importáncia do consumo excessivo de álcool como problema subjacente de doentes assistidos em hospitais ${ }^{18,22}$. No presente trabalho não foram utilizados questionários padronizados para identificação de pacientes alcoolistas ${ }^{15}$, mas buscou-se comparar nas populações estudadas parâmetros qualitativos e quantitativos de consumo de álcool.

$A$ associação entre paracoccidioidomicose e consumo mais elevado de álcool foi estabelecida para a forma crônica da infeç̧ão, na qual $50 \%$ dos doentes ingeriam acima de $60 \mathrm{ml}$ por dia, verificando-se também preferência mais acentuada por aguardente de cana, bebida de elevado teor alcoólico. Os valores relacionados na Tabela 2 permitiram estimar em 2,3 o risco relativo ${ }^{9}$ de aquisição de paracoccidioidomicose crônica para aqueles com consumo etílico médio superior a $60 \mathrm{ml}$ por dia. Nos doentes mais jovens, com a forma aguda/subaguda da infecção, evidenciou-se consumo excessivo do álcool apenas esporadicamente.

A predisposição de alcoolistas adquirirem micoses profundas já foi observada anteriormente em relação a candidíase ${ }^{1}$, criptococose $\mathrm{e}^{21}$, esporotrico$\mathrm{se}^{16} \mathrm{e}$ aspergilose ${ }^{8}$. O etilismo causa desnutrição e reduz a defesa imunológica do organismo, principalmente dos doentes com doença hepática alcoólica, referindo-se que a partir de $50 \mathrm{~g} / \mathrm{dia}$ o risco de evolução para cirrose seria independente da dose de álcool ingerida ${ }^{14}$. Dos efeitos imunossupressores do álcool, a redução na imunidade celular foi relacionada com a maior incidência de tuberculose entre alcoolistas ${ }^{17}$ e provavelmente também predispõe a outras infecções granulomatosas, inclusive a paracoccidioidomicose. Porém, a desnutrição foi a provável causa da maior gravidade da paracoccidioidomicose em ratos alcoolizados e infectados experimentalmente ${ }^{13}$.

$O$ etilismo parece ter maior importância na patogenia da forma crônica da micose, cujos doentes consomem mais álcool e por tempo mais prolongado do que os pacientes com a forma aguda. O álcool reduz a função mucociliar e a produção de surfactante ${ }^{2}$, podendo lesar a mucosa das vias respiratórias ao ser transformado em acetaldeído pela flora bacteriana superficial ${ }^{19}$. É possivel que tais efeitos estejam contribuindo para a grande frequiência de lesão pulmonar e das vias respiratórias superiores na forma crônica da paracoccidioidomicose. O tratamento desses doentes também poderia estar sendo prejudicado pelo etilismo, a julgar pela tendência observada de reativação mais frequiente da moléstia entre os grandes bebedores (ingestão superior a $100 \mathrm{ml} / \mathrm{dia}$ ).
Concluindo, os dados obtidos sugerem que o consumo excessivo de álcool seja fator predisponente da paracoccidioidomicose e que talvez possa prejudicar a cura dos doentes com a forma crônica da infecção, os quais devem ser alertados a este respeito. A doença resulta geralmente em pneumopatia crônica incapacitante e poderia ser relacionada entre as consequências indiretas do alcoolismo, sobretudo em trabalhadores rurais da América Latina.

MARTINEZ, R. \& MOYA, M.J. [The relationship between paracoccidioidomycosis and alcoholism] Rev. Saúde públ., S. Paulo, 26: 12 - 6, 1992. The relationship between alcoholism and paracoccidioidomycosis was evaluated by the casecontrol method. The alcohol consumption of 4 groups of patients was compared: 50 patients with chronic paracoccidioidomycosis, 20 patients with the acute or subacute form of this mycosis and their respective control groups of hospitalized patients, each case matched by sex and age. Between September 1986 and July 1988 the cases and their controls were interviewed by one and the same investigator using a questionnaire on drinking habits: quantity and type of beverage consumed, time of onset and frequency of use and whether they had manifested symptoms of inebriation or of alcohol dependence previously. As compared with control patients, the mean daily ingestion of alcohol in excess of $60 \mathrm{ml}$ was more frequent in the chronic paracoccidioidomycosis group $(50.0 \% \mathrm{x}$ $30.0 \%)$. These patients also preferred to drink sugar cane brandy more frequently $(89.4 \% \times$ $68.3 \%$ ). When the average daily consumption of ethyl alcohol exceeded $100 \mathrm{ml}$, most patients presented a recurrence of infection during or after antifungal therapy. In the acute-subacute paracoccidioidomycosis group, $64.3 \%$ of the patients reported inebriation on one or more occasions, versus $17.6 \%$ in the respective control group. The results suggest that alcoholism can be a predisposing factor to paracoccidioidomycosis and, probably, accounts for a worse prognosis for this infection.

Keywords: Alcoholism, complications. Paracoccidioidomycosis, complications.

\section{Referências Bibliográficas}

1.BARDWELL, A.; HILL, D.W.; RUNYON, B.A.; KOSTER, F.T. Disseminated macronodular cutaneous candidiasis in chronic alcoholism. Arch. intern. Med, 146: 385-6, 1986.

2.BOMALASKI, J.S. \& PHAIR, J.P. Alcohol, immunossupression, and the lung. Arch. intern. Med, 142: 2073-4, 1982.

3.CARON-RUFFINO, M. \& RUFFINO-NETTO, A. Associação entre alcoolismo e tuberculose pulmonar. Rev. Saúde públ., S. Paulo, 13: 183-94, 1979. 
4.COLOMBO, A.; FORJAZ, M.H.H.; FISCHMAN, O.; SALOMÃO, R.; CALVO MORALES, B.; LONGO, J.C. Epidemiological study of paracoccidioidomycosis infection in São Paulo. In: International Congress for Infectious Diseases, Rio de Janeiro, 1988. Abstract book. Rio de Janeiro, 1988. p. 86.

5.DANIEL, W.W. Applied nonparametric statistics. Boston, Houghton Mifflin Company, 1978.

6.DESAI, I.D.; TAVARES, M.L.G.; OLIVEIRA, B.S.D.; DOUGLAS, A.; DUARTE, F.A.M.; DUTRA DE OLIVEIRA, J.E. Food habits and nutritional status of agricultural migrant workers in Southern Brazil. Amer. J. clin. Nutr., 33: 702-14, 1980.

7.DIXON, WJ.\& MASSEY Jr., F.J. Intraduction to statistical analysis. 31 rd ed. Tokyo, Mc-Graw-Hill Kogakusha Ltd., 1969.

8.D'SILVA, H.; BURKE Jr.; J.F.; CHO, S.Y. Disseminated aspergillosis in a presumably immunocompetent host. J.Amer. med Ass., 248: 1495-7, 1982.

9.FORATTINI, O.P. Epidemiologia geral. São Paulo, Ed. Edgar Blücher/Ed. USP, 1976.

10.FRANCO, M. Host-parasite relationship in paracoccidioidomycosis. J. med. Vet. Mycol., 25: 5-18, 1987.

11. FRANCO, M.; MONTENEGRO, M.R.; MENDES, R.P.; MARQUES, S.A.; DILLON, N.L.; MOTA, N.G.S. Paracoccidioidomycosis: a recently proposed classification of its clinical forms. Rev. Soc. bras. Med. trop., 20: 129-32, 1987.

12.FRIEDMAN, L.N.; SULLIVAN, G.M.; BEVILAQUA, R.P.; LOSCOS, R. Tuberculosis screening in alcoholics and drug addicts. Amer. Rev. respir. Dis., 136: 1188-92, 1987.

13. GOLDANI, L.Z;; VANNUCCHI, H.; ZUCOLOTO, S.; MARTINEZ, R. Experimental paracoccidioidomycosis in ethyl alcohol-fed rats. Braz, J. med. Biol. Res., 24: $901-8,1991$.

14.JOHNSON, R.D. \& WILLIANS, R. Immune responses in alcoholic liver disease. Alcohol clin. exp. Res., 10: $471-86,1986$.
15. JORGE, M.R. O diagnóstico do alcoolismo: entrevistas estruturadas, questionários e exames de laboratório. In: Ramos, S. de P. \& Bertolote, J.M. Alcoolismo hoje. $2^{2}$ ed. Porto Alegre, Ed. Artes Médicas, 1990. p. 63-74.

16.LYNCH, P.J.; VOORHEES, J.J.; HARRELL, E.R. Systemic sporotrichosis. Ann. intern. Med., 73: 23-30, 1970 .

17.MACGREGOR, RR Alcohol and immune defense. $J$. Amer. med. Ass., 256: 1474-9, 1986.

18.MASUR, J.\& JORGE, M.R. Dados relacionados a bebidas alcoólicas e alcoolismo no Brasil: uma revisão. Rev. Ass.bras. Psiq., 8: 157-65, 1986.

19.MIYAKAWA, H.; BARAONA, E; CHANG, J.C.; LESSER, M.D.; LIEBER, C.S. Oxidation of ethanol to acetaldehyde by bronchopulmonary washings: role of bacteria. Alcohol clin. exp. Res., 10: 517-20, 1986.

20.PERSON, J.\& MAGNUSSON, P.-H. Comparison between different methods of detecting patients with excessive consumption of alcohol. Acta medscand. 223: $101-9,1988$.

21.PINCUS, M.R.; SILVA-HUTNER, M.; REBATTA, G.; BRANWOOD, A.W. Disseminated cryptococcosis in an asymptomatic alcoholic man. Arch.intern. Med. 141: 796-7, 1981.

22. ZUARDI, A.W.; CORREA, A.J.; CACERES-VERA, P.E.F.; DEL LAMA, J.; FERRAZ, C.A. Valor do questionário "CAGE" na detecção precoce dos pacientes com risco para o desenvolvimento de sindrome de abstinência do álcool, num hospital geral. Rev. Ass. bras. Psiq., 9: 157-60, 1987.

Recebido para publicação em 28/1/1991

Reapresentado em 2/1/1992

Aprovado para publicação em $6 / 1 / 1992$ 